\title{
The scope and processing of for-adverbials: A reply to Deo and Piñango*
}

\author{
Lucas Champollion \\ New York University
}

\begin{abstract}
Deo \& Piñango (2011) propose a novel account of measure adverbials like for an hour that focuses on their puzzling scopal behavior, their ability to shift predicates into iterative interpretations, and the higher processing cost that this shift engenders. The scopal behavior, though not the processing cost, had been previously modeled in classical accounts of for-adverbials (e.g., Dowty 1979; Krifka 1998). Unlike these accounts, D\&P do not provide a semantic explanation of the aspectual sensitivity of for-adverbials. I argue that only a synthesis of D\&P and classical accounts captures the full empirical picture. This synthesis both restores the explanation of the aspectual sensitivity, and improves on D\&P's account of the scopal behavior. It also opens the door to an explanation of data which suggests that the processing cost of iterativity is not uniform, but varies according to the algebraic properties of the underlying predicates.
\end{abstract}

Keywords: aspect, algebraic semantics, coercion, for-adverbials, iterativity, scope

\section{Introduction}

Deo \& Piñango (2011), called D\&P here for short, present an ambitious theory of for-adverbials that focuses on their puzzling scopal behavior, their ability to shift predicates into iterative interpretations, and the higher processing cost that this shift engenders. The scopal behavior, though not the processing cost, had been previously modeled in classical accounts of for-adverbials (Dowty 1979; Krifka 1998), which focus on their aspectual sensitivity, that is, their ability to distinguish between atelic predicates as in (1a) and telic ones as in (1b):

(1) a. John ran / drove towards the store / drank wine for an hour.

b. ?John ran a mile / drove to the store / drank a glass of wine for an hour.

* For comments and discussion about this work, I thank Chris Barker, Dylan Bumford, and the SALT reviewers, as well as audiences at SALT 23, at the Stuttgart 2011 workshop on aspect and modality in lexical semantics, and at the Göttingen 2011 Workshop "Indefinites and Beyond". Special thanks to Ashwini Deo and Maria Mercedes Piñango for helpful discussion and comments and for their positive attitude towards this project. All errors are mine. 
The scope and processing of for-adverbials

For-adverbials are commonly considered the most reliable diagnostic of the distinction between these predicates (Vendler 1957; Verkuyl 1989). For this reason, the exact truth conditions of for-adverbials bear on the question of what formal properties best capture telicity and atelicity. Classical accounts typically focus on investigating such properties as quantization or the subinterval property in detail (e.g., Krifka 1998). But in contrast to these accounts, D\&P choose not to provide a semantic explanation of the aspectual sensitivity of for-adverbials.

In this paper, I argue that only a synthesis of D\&P and classical accounts captures the full empirical picture. This synthesis both restores the explanation of the aspectual sensitivity, and improves on D\&P's account of the scopal behavior. It also opens the door to an explanation of data which suggests that the processing cost of iterativity is not uniform, but varies according to the algebraic properties of the underlying predicates.

For my synthesis, I will draw on a combination of previous accounts which have each addressed a part of the empirical picture discussed in D\&P 2011 (Dowty 1979; Krifka 1998; van Geenhoven 2004; Kennedy 2012; Champollion 2010a,b). For ease of comparison, I will present my proposal as a series of incremental changes to D\&P 2011 that incorporate the relevant insights from these previous accounts, rather than choosing the system of any of these accounts as a starting point. As far as I can tell, the changes to D\&P 2011 that I propose here seem to be backwards compatible with the analysis of generic and habitual sentences in Deo 2009 from which the account is technically derived.

Let me start by reviewing the facts that D\&P's theory of for-adverbials sets out to account for. The first fact concerns iterative interpretations. For-adverbials may cause predicates to be interpreted iteratively (Egg 1994, 1995; van Geenhoven 2004, 2005):

(2) a. Mary biked to the store for two months.

b. The girl dove into the pool for an hour.

The predicates bike to the store and dive into the pool are telic on their noniterative interpretations. However, for-adverbials not only trigger iterative interpretations of telic predicates. So-called semelfactive predicates like jump (Smith 1997) are atelic to begin with, but for-adverbials can still cause them to be interpreted iteratively:

(3) a. The horse jumped for an hour.

As reviewed by Deo \& Piñango 2011, iterative interpretations engender cost by various psycho- and neurolinguistic measures: increased centro-parietal activity (Downey 2006); increased reading times and brain activity (Brennan \& Pylkkänen 
2008); longer reaction time in cross-modal lexical decision (Piñango, Zurif \& Jackendoff 1999; Piñango, Winnick, Ullah \& Zurif 2006); and comprehension difficulties in Wernicke's Aphasics (Piñango \& Zurif 2001).

For-adverbials can also trigger other kinds of reinterpretations when they combine with telic predicates (Moens \& Steedman 1988). For example, in some cases they can specify the duration of the event's consequent or result state instead of the duration of the event to which the predicate itself applies (Dowty 1979; Piñón 1999):

(4) John opened the window for five minutes.

Another example is partitive reinterpretation, which can occur when the interval introduced by the for-adverbial is too short to make an iterative interpretation plausible:

a. Mary read a book for an hour.

b. Mary baked a cake for twenty minutes.

The availability of a partitive interpretation can in some cases be blocked by an explicit endpoint description, as in resultatives (Smollett 2005):

a. Mary polished the countertop for 15 minutes.

b. *Mary polished the countertop smooth for 15 minutes.

c. *Mary polished the countertop to a shine for 15 minutes.

As Ashwini Deo (p.c.) points out to me, not all explicit endpoint descriptions block for-adverbials, as shown by the following web-attested example:

(7) I cooked the roux to a nice toffee brown for about 10 minutes while chopping my onions, celery and carrot. (http://www.chronicmastication.com/2009/02/ refrigerator-gumbo.html)

In this kind of case, the for-adverbial indicates the time required for the event to be realized to the specified degree. Specifically, it is not true that the predicate cook the roux to a nice toffee brown holds at both the larger interval and subintervals of that interval (one of the key components of the analysis presented above as well as the traditional accounts). As far as I can tell, it is an open question in all theories of aspectual composition why the endpoint description to a nice toffee brown does not force a telic interpretation in the way to a shine does. I will not be able to answer this question here. In any case, the main point is that the partitive interpretation is not always available. Thus, not all predicates can undergo this reinterpretation process.

Let me now describe the puzzling scopal behavior of for-adverbials. As has been known since Carlson 1977 and as has been more recently discussed by Kratzer (2007), indefinites in the syntactic scope of for-adverbials are unable to covary with 
The scope and processing of for-adverbials

the for-adverbial in the way that they can covary with a universal quantifier when they occur in its syntactic scope. For example, the following sentences from Kratzer 2007 all involve reference to a single cart, a single phone number, and so on:

a. John pushed a cart for an hour.

b. I dialed a wrong phone number for five minutes.

c. She bounced a ball for 20 minutes.

d. He kicked a wall for a couple of hours.

e. She opened and closed a drawer for half an hour.

f. I petted a rabbit for two hours.

Besides singular indefinites, other DPs such as numerals also lack a covarying reading. For example, (9) involves reference to a set of thirty zebras that does not change throughout the three hours in question:

(9) John saw thirty zebras for three hours.

This lack of covariation is surprising if for an hour is interpreted as at each moment of an hour, as is the case in many theories of for-adverbials. One might try to account for these facts by stipulating obligatory QR of the indefinite above the for-adverbial, as suggested by Krifka (1998). But as observed by Kratzer (2007), the phenomenon can also be observed in German, which is otherwise known for having a preference for surface scope. Thus each of the two sentences in (10) must involve reference to a single phone number:

a. Ich hab' fünf Minuten lang eine falsche Telefonnummer gewählt. I have five minutes long a wrong telephone.number dialed.

b. Ich hab' eine falsche Telefonnummer fünf Minuten lang gewählt. I have a wrong telephone.number five minutes long dialed.

And as noted by Zucchi \& White (2001), the effect does not occur when a universal quantifier like every day intervenes, as in (11). Here, the fleas covary:

(11) John found a flea on his dog every day for a month.

These facts suggest that the lack of covariation is not likely to be due to obligatory QR of the indefinite above the for-adverbial.

The ban against covariation even holds when plausibility considerations would favor a reading where the indefinite covaries. Example (12) is taken from Zucchi \& White 2001:

??John found a flea on his dog for a month. 
The examples in (13) are taken from D\&P 2011:

a. ??John noticed a discrepancy/two discrepancies for a week.

b. ??John discovered a new proof/two new proofs for a week.

Once the lack of covariation is explained, the oddness of these examples can be attributed to the fact that plausibility facts disfavor an interpretation where there is no covariation. For example, fleas are typically not found repeatedly, discrepancies are typically not noticed repeatedly, and so on.

The behavior just described does not hold across the board for all types of noun phrases. Bare plurals and mass nouns do not have to take distributive wide scope over for-adverbials (Carlson 1977; Verkuyl 1972; Dowty 1979):

a. John found fleas on his dog for a month.

b. John discovered crabgrass in his yard for six weeks.

a. Tourists discovered that quaint little village for years.

b. Water leaked through John's ceiling for six months.

Covariation is also possible when a salient level of granularity can be inferred from context. The notion of a contextually determined partition originates independently in Moltmann (1991) and in Deo (2009). It is further discussed in Champollion (2010b) and in D\&P. To take an example from the latter, in a context where the daily pill intake of patients is salient (in a hospital, for example), sentence (16) is licit despite the fact that it does not require any pill to be taken more than once. And D\&P's example (17) is understood as involving reference to several snowmen, presumably because world knowledge makes the cycle of seasons salient here:

(16) The patient took two pills for a month and then went back to one pill.

(17) We built a huge snowman in our front yard for several years.

Covarying singular indefinites take extra time to process compared with bare plurals. D\&P discuss the fact that reading time increases at the for-adverbial in (18a) compared with (18b) in self-paced reading tests conducted by Todorova, Straub, Badecker \& Frank (2000). Note that (18a) involves a covarying indefinite.

(18) a. Even though Howard sent a large check to his daughter for many years, she refused to accept his money.

b. Even though Howard sent large checks to his daughter for many years, she refused to accept his money.

To summarize, D\&P set out to explain the following facts: For-adverbials trigger iterative interpretations; these iterative interpretations engender processing costs; 
The scope and processing of for-adverbials

they trigger partitive interpretations on some accomplishments; they are unable to cause indefinites and numerals to covary, except for salience and world knowledge effects; and they are fine with letting bare plurals and mass nouns covary.

The rest of this paper is as follows. In Section 2, I present D\&P's system. Section 3 argues that their system does not account for some of the facts just mentioned. Section 4 presents my synthesis of their system and algebraic accounts. The scopal behavior of for-adverbials is discussed in Section 5. Section 6 concludes.

\section{The account in Deo \& Piñango 2011}

Let me start with some notation and background notions. I use lowercase $i, j, \ldots$ for moments (intervals of infinitesimal length) and capital $I, J, \ldots$ for intervals in general. I write $\sqsubseteq$ for "is a subinterval of" (a reflexive relation) and $\sqsubset$ for "is a proper subinterval of" (an irreflexive relation). I write $\tau$ for the runtime function. I write $\operatorname{AT}(P, I)$ to generalize over event and interval predicates in the following way:

$$
\operatorname{AT}(P, I)= \begin{cases}P(I) & \text { if } \mathrm{P} \text { is an interval predicate } \\ \exists e[P(e) \wedge I=\tau(e)] & \text { if } \mathrm{P} \text { is an event predicate }\end{cases}
$$

I assume that atelicity and telicity correspond to two higher-order properties: the subinterval property and (temporal) quantization. This is an idealization - for more discussion, see Champollion 2010b - but it will do for the purpose of this paper. In particular, I ignore here the minimal-parts problem that arises with very short runtimes (Dowty 1979). This is justified because the minimal-parts problem has nothing to do with the problems discussed in this paper.

The subinterval property is usually attributed to Bennett \& Partee (1972), though they discuss only the concept and do not use this term. A predicate has the subinterval property just in case whenever it holds at an interval I, it holds at every subinterval of I, as defined in (20). For example, the atelic predicate run has the subinterval property, since it licenses inferences like (21).

$$
\begin{aligned}
& \text { Subinterval(P) } \stackrel{\text { def }}{=} \forall I[\mathrm{AT}(P, I) \rightarrow \forall J[J \sqsubset I \rightarrow \mathrm{AT}(P, J)]] \\
& \text { John ran from } 3 \text { to } 5 \mathrm{pm} . \Rightarrow \text { John ran from } 3 \text { to } 4 \mathrm{pm} .
\end{aligned}
$$

A predicate is temporally quantized just in case whenever it holds at an interval, it does not hold at any one of its subintervals. The definition in (22) is based on the definition of quantization in Krifka 1986. Temporal quantization is easier to work with than quantization and will do for the present purposes. For example, the telic predicate run a mile is temporally quantized since it blocks inferences like (23).

$$
\text { Temporally-quantized(P) } \stackrel{\text { def }}{=} \forall I[\mathrm{AT}(P, I) \rightarrow \forall J[J \sqsubset I \rightarrow \neg \mathrm{AT}(P, J)]]
$$


Let us now examine D\&P's analysis of for-adverbials, which is based on the notion of regular partitions. A regular partition of an interval $I$, written $\mathscr{R}_{I}$, is a set of disjoint intervals of equal length whose concatenation equals $I$, as in the following picture:

D\&P write $\mathscr{R}_{I}^{c}$ for a contextually determined regular partition of $I$, and $\mathscr{R}_{I}^{\text {inf }}$ for a regular partition of $I$ whose intervals have infinitesimal value (that is, they are moments). D\&P write COIN(P,I) to generalize over intervals and events, as in (24). Note that unlike AT, COIN allows overlap (written $\circ$ ) if $\mathrm{P}$ is an event predicate:

$$
\operatorname{CoIN}(\mathrm{P}, \mathrm{I})= \begin{cases}P(I) & \text { if } \mathrm{P} \text { is an interval predicate } \\ \exists e[P(e) \wedge I \circ \tau(e)] & \text { if } \mathrm{P} \text { is an event predicate }\end{cases}
$$

D\&P translate a for-adverbial as a universal quantifier over a regular partition:

$$
[[\text { for an hour }]]_{D \& P}=\lambda P \lambda I\left[\operatorname{hours}(I)=1 \wedge \forall J\left[J \in \mathscr{R}_{I}^{c} \rightarrow \operatorname{COIN}(P, J)\right]\right]
$$

In D\&P's system, predicates to which for-adverbials apply are always event predicates, except when they already contain another aspectual modifier. Since the latter case does not occur in any of the examples they discuss, we can rewrite (25) for the purpose of exposition as follows:

$$
[[\text { for an hour }]]_{D \& P}=\lambda P \lambda I\left[\operatorname{hours}(I)=1 \wedge \forall J\left[J \in \mathscr{R}_{I}^{c} \rightarrow \exists e[P(e) \wedge J \circ \tau(e)]\right]\right]
$$

D\&P use the infinitesimal-cell-length partition $\mathscr{R}_{I}^{\text {inf }}$ to model continuous (that is, non-iterative) readings of for-adverbials. This amounts to universal quantification over moments. In this case, we can rewrite $\circ$ as $\sqsubseteq$ since we are dealing with moments and since any moment that overlaps with an interval is contained in it.

$$
\begin{aligned}
& {[[\text { for an hour }]]_{D \& P}^{\text {continuous }}} \\
& =\lambda P \lambda I\left[\operatorname{hours}(I)=1 \wedge \forall i\left[i \in \mathscr{R}_{I}^{\text {inf }} \rightarrow \exists e[P(e) \wedge i \circ \tau(e)]\right]\right] \\
& =\lambda P \lambda I[\operatorname{hours}(I)=1 \wedge \forall i[i \sqsubset I \rightarrow \exists e[P(e) \wedge i \sqsubseteq \tau(e)]]]
\end{aligned}
$$

This entry is used to model sentences that do not involve iterativity. For example, John walked for an hour is predicted to be true of any one-hour timespan of which every moment is temporally contained in an event of John walking:

$$
\begin{aligned}
& {[[\text { John walk for an hour }]] \text { D\&P }} \\
& =\lambda I[\text { hours }(I)=1 \wedge \forall i[i \sqsubset I \rightarrow \exists e[[[\text { John walk }]](e) \wedge i \sqsubseteq \tau(e)]]]
\end{aligned}
$$


The scope and processing of for-adverbials

D\&P assume that iterative readings arise when the interval is partitioned into subintervals which are relatively small-sized compared with the interval of the foradverbial, but still larger than moments. This is illustrated for accomplishments in (29) and for semelfactives in (30). The first sentence is predicted to be true of any one-month timespan if each cell of its contextually given regular partition overlaps with an event of Mary biking to the store. The second sentence is predicted true of any one-hour timespan if each cell of its contextually given regular partition overlaps with an event of the horse jumping.

$[[\text { Mary bike to the store for a month }]]_{D \& P}^{\text {iterative }}$

$=\lambda I\left[\operatorname{months}(I)=1 \wedge \forall J\left[J \in \mathscr{R}_{I}^{c} \rightarrow \exists e[[[\right.\right.$ Mary bike to the store $]](e) \wedge J \circ$ $\tau(e)]]]$

$[[\text { The horse jump for an hour }]]_{D \& P}^{\text {iterative }}$

$=\lambda I\left[\operatorname{hours}(I)=1 \wedge \forall J\left[J \in \mathscr{R}_{I}^{c} \rightarrow \exists e[[[\right.\right.$ The horse jump $\left.\left.]](e) \wedge J \circ \tau(e)]\right]\right]$

The value of the variable $c$ that determines the size of the cells of the partition is anaphoric on the context. In this respect, D\&P follow the approach developed in Deo 2009 (see also Moltmann 1991 and Champollion 2010b for similar ideas). They attribute the higher processing costs for iterative interpretations to the process of retrieving a value for $c$ from the context, and they assume that this process is not necessary when $c$ can be set to an infinitesimal value. In this way, they capture the fact that continuous readings do not lead to extra processing costs.

\section{Critique of D\&P's account}

In this section, I offer a critique of D\&P's account. First, note that D\&P do not model the aspectual sensitivity of for-adverbials. To see this, let $P$ be a temporally quantized (and therefore atelic) event predicate that holds at some interval $I$ of at

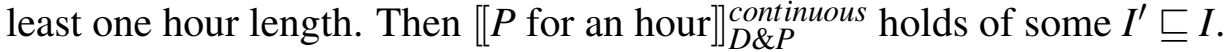

Proof: By assumption, we have hours $(I) \geq 1 \wedge \mathrm{AT}(P, I)$. Using the definition of AT, we expand this into hours $(I) \geq 1 \wedge \exists e[P(e) \wedge I=\tau(e)]$. Since hours is an extensive measure function, this is equivalent to $\exists I^{\prime}\left[I^{\prime} \sqsubseteq I \wedge\right.$ hours $\left(I^{\prime}\right)=1 \wedge \exists e\left[P(e) \wedge I^{\prime} \sqsubseteq\right.$ $\tau(e)]]$, which is equivalent to $\exists I^{\prime}\left[I^{\prime} \sqsubseteq I \wedge\right.$ hours $\left(I^{\prime}\right)=1 \wedge \forall i\left[i \sqsubset I^{\prime} \rightarrow \exists e[P(e) \wedge i \sqsubseteq\right.$ $\tau(e)]]$ by transitivity of temporal inclusion. Now it follows from D\&P's entry for for an hour shown in (27) that $\exists I^{\prime}\left[I^{\prime} \sqsubseteq I \wedge[[P \text { for an hour }]]_{D \& P}^{\text {continuous }}\left(I^{\prime}\right)\right]$. QED.

The nature of the problem is that when we apply D\&P's for-adverbial to a subinterval $I^{\prime}$ of $I$, it does not check whether $P$ holds at that subinterval. All it does is make sure that each moment of $I^{\prime}$ is contained in an interval at which $P$ holds. By assumption, $I$ itself is such an interval.

To be sure, D\&P's non-modeling of the aspectual sensitivity of for-adverbials is 
not an oversight but a design decision. In their view, "telic predicates are perfectly acceptable with for-adverbs" since they can give rise to iterative and partitive interpretations. They explicitly reject the idea that telic predicates must be coerced into atelic predicates before combining with a for-adverbial. D\&P expect that telic predicates are ruled out by pragmatic considerations, though they do not describe these pragmatic considerations for the continuous case. I come back to this point in Section 6. For now, let us set the aspectual sensitivity aside and discuss the other empirical facts laid out above.

I start with partitive reinterpretations. We have seen that predicates like polish the countertop and cook the roux to a nice toffee brown can get such interpretations, but not others like polish the countertop smooth and polish the countertop to a shine. An account of for-adverbials should therefore allow certain accomplishments to lend themselves to partitive reinterpretation why allowing others to resist it. Since D\&P make the infinitesimal partition length available at no cost, they predict partitive-like interpretations for all accomplishments. Let us first look at a case that is treated correctly. The following sentence is predicted to be acceptable, and true of any fifteen-minute timespan of which every moment is temporally contained in an event of Mary polishing the countertop.

$[[\text { Mary polish the countertop for } 15 \text { minutes }]]_{D \& P}^{\text {continuous }}$

$=\lambda I[\operatorname{minutes}(I)=15 \wedge \forall i[i \sqsubset I \rightarrow$

$\exists e[[[$ Mary polish the countertop $]](e) \wedge i \sqsubseteq \tau(e)]]]$

Since nothing stops continuous for-adverbials from applying to accomplishments with explicit endpoint descriptions, the following sentence is predicted to be acceptable as well, and to be true of any fifteen-minute timespan of which every moment is temporally contained in an event of Mary polishing the countertop smooth. However, we would expect this sentence to be ruled out, as shown in (6).

$[[\text { Mary polish the countertop smooth for } 15 \text { minutes }]]_{D \& P}^{\text {continuous }}$

$=\lambda I[\operatorname{minutes}(I)=15 \wedge \forall i[i \sqsubset I \rightarrow$

$\exists e[[[$ Mary polish the countertop smooth $]](e) \wedge i \sqsubseteq \tau(e)]]]$

Moving on to the scopal behavior of indefinites, D\&P's account overgenerates readings in which indefinites covary, since they are able to take scope under the universal of the for-adverbial. For example, sentence (33) is predicted to be true of any one-hour timespan of which every moment is temporally contained in an event of John pushing a cart. In (33), the quantifier $\forall i$ that introduces moments takes scope over the quantifier $\exists x$ that introduces carts, so these moments can involve different carts. Imagine for example that John pushed a certain cart from $3 \mathrm{pm}$ to $3: 30 \mathrm{pm}$ and then another cart from $3: 30 \mathrm{pm}$ to $4 \mathrm{pm}$. Then (33) is incorrectly predicted to be true 
The scope and processing of for-adverbials

of the interval from 3 to $4 \mathrm{pm}$, since at any moment within it, John pushed a cart.

[John push a cart for an hour $]_{D \& P}^{\text {continuous }}$

$=\lambda I[\operatorname{hours}(I)=1 \wedge \forall i[i \sqsubset I \rightarrow \exists e[[[$ John push a cart $]](e) \wedge i \sqsubseteq \tau(e)]]]$

$=\lambda I[\operatorname{hours}(I)=1 \wedge \forall i[i \sqsubset I \rightarrow \exists e \exists x[\operatorname{cart}(x) \wedge \operatorname{push}(e$, john, $x) \wedge i \sqsubseteq \tau(e)]]]$

The same problem extends to iterative readings. Recall that D\&P model them via anaphorically retrieved partitions, for example partitions of weeks into days. Consider again sentence (12), repeated here:

??John found a flea on his dog for a month.

If an out-of-the-blue context provided an antecedent $c$ for such a partition in the case of this sentence, it would be incorrectly predicted to be acceptable. It would be true of any week whose days each overlap with an event of John finding a flea, even if different fleas are found, as shown below:

$$
\begin{aligned}
& {[[\text { John find a flea (on his dog) for a month }]]_{D \& P}^{\text {iterative }}} \\
& =\lambda I\left[\text { months }(I)=1 \wedge \forall J\left[J \in \mathscr{R}_{I}^{c} \rightarrow \exists e\left[\left[\left[\mathrm{John}_{\text {find a flea }]}\right](e) \wedge J \circ \tau(e)\right]\right]\right]\right. \\
& =\lambda I\left[\operatorname{months}(I)=1 \wedge \forall J\left[J \in \mathscr{R}_{I}^{c} \rightarrow \exists e \exists x[\text { flea }(x) \wedge \text { find }(e, \text { john, } x) \wedge J \circ \tau(e)]\right]\right]
\end{aligned}
$$

So we need to conclude that an out-of-the-blue context does not provide such an antecedent. But this conclusion incorrectly rules out sentence (14a), repeated here:

$[[\text { John find fleas (on his dog) for a month }]]_{D \& P}^{\text {iterative }}$

$$
\begin{aligned}
& =\lambda I\left[\operatorname{months}(I)=1 \wedge \forall J\left[J \in \mathscr{R}_{I}^{c} \rightarrow \exists e[[[\text { John find fleas }](e) \wedge J \circ \tau(e)]]\right]\right. \\
& =\lambda I\left[\operatorname{months}(I)=1 \wedge \forall J\left[J \in \mathscr{R}_{I}^{c} \rightarrow \exists e \exists X[\text { fleas }(X) \wedge \text { find }(e, \text { john }, X) \wedge J \circ\right.\right. \\
& \tau(e)]]]
\end{aligned}
$$

Assuming the same partition as in (34) above, this sentence is predicted true of any one-month timespan if each of its days overlaps with an event of John finding fleas. D\&P's account does not capture the contrast between such sentences because, unlike classical accounts such as Krifka 1998, it is not sensitive to the algebraic properties of the verb phrase - in this case, to the distinction between a flea and fleas. The fact that telic predicates like find a flea are temporally quantized, while atelic ones like find fleas and push a cart are not, does not enter the picture. This also means that D\&P's account does not explain the finding by Todorova et al. (2000) that singular indefinites take longer reading time than bare plurals (as discussed above). And for the same reason, D\&P cannot explain contrasts between pairs with singular indefinites where one is better than the other, and corresponding pairs with bare plurals where both are equally acceptable:

a. ?John found a flea on his dog for a month. =(12) 
b. We built a huge snowman in our front yard for several years. = (17)

a. John found fleas on his dog for a month. $=(14 \mathrm{a})$

b. We built huge snowmen in our front yard for several years.

D\&P cannot account for the contrast between (37a) and (37b) by stipulating that the former cannot access a contextually given partition, because this would also predict a contrast between (38a) and (38b), where there is none.

\section{Synthesizing D\&P and algebraic accounts}

From the above observations, I derive the following desiderata for a synthesized account. We would like to reintroduce the sensitivity of for-adverbials to the algebraic properties of their predicates, not only because this helps model their aspectual sensitivity, but also because, as we have seen, certain scope and processing facts appear to be related to these algebraic properties. While we would like to keep D\&P's ability to account for the availability of iterative interpretations, we would also like to capture the added processing load engendered by these interpretations. The fact that for-adverbials trigger partitive interpretations on some accomplishments should be modeled, as well as the fact that overt endpoints sometimes block these partitive reinterpretations. And finally, we should correctly account for the scope facts involving indefinites and numerals.

The following treatment is based on insights in Kratzer 2007 and in Champollion 2010b. Rather than introducing these accounts from scratch, I will show how to modify D\&P's account to get equivalent effects. Let us start with D\&P's translation of a for-adverbial, repeated below from (25).

$$
[[\text { for an hour }]]_{D \& P}=\lambda P \lambda I\left[\operatorname{hours}(I)=1 \wedge \forall J\left[J \in \mathscr{R}_{I}^{c} \rightarrow \operatorname{COIN}(P, J)\right]\right]
$$

To reintroduce sensitivity to the telic/atelic distinction, we add a conjunct that says that the predicate applies at the whole hour, and we replace COIN by AT. Remember that for event predicates, CoIN allows temporal overlap, while AT does not. So the runtime of the event is now required to be equal to the universally quantified interval $J$. The result is shown in (40). I call this entry "step 1" because I will introduce another change later.

$$
[[\text { for an hour }]]_{\text {step } 1}=\lambda P \lambda I\left[\operatorname{AT}(P, I) \wedge \operatorname{hours}(I)=1 \wedge \forall J\left[J \in \mathscr{R}_{I}^{c} \rightarrow \operatorname{AT}(P, J)\right]\right]
$$

The added conjunct in (40) prevents for-adverbials from combining with any quantized predicate $P$, since such a predicate cannot satisfy AT(P,I) and AT(P,J) at the same time (assuming $J \neq I$ ). This restores the sensitivity of for-adverbials to the algebraic properties of the predicate. As long as $\mathscr{R}_{I}^{c}$ has at least two cells, 
The scope and processing of for-adverbials

for-adverbials will not be able to truthfully combine with any temporally quantized predicate.

Proof: Let $P$ be a temporally quantized predicate. Suppose counterfactually that $\left[[P \text { for an hour }]_{\text {step } 1}\right.$ holds of some interval $I$. Then $P$ holds at $I$ and at every $J$ such that $J \in \mathscr{R}_{I}^{c}$. Take one of these $J$ s. By definition of $\mathscr{R}_{I}^{c}, J$ is a subinterval of $I$. Since $\mathscr{R}_{I}^{c}$ has at least two cells and since they are disjoint, $J$ must be a proper subinterval of $I$. Since $P$ is temporally quantized, it cannot hold both at $J$ and at $I$. Contradiction.

The added conjunct requires that $P$ hold at the whole hour. At first sight, this means we can no longer account for iterative readings of semelfactives. For example, (41) is predicted to be true of any one-hour long interval such that the horse jumped at that hour (and also at each point within that hour). But how can a single jump last an entire hour?

[[The horse jump for an hour $]]_{\text {step } 1}$

$=\lambda I[\exists e[\operatorname{jump}(e$, the horse $) \wedge I=\tau(e) \wedge \operatorname{hours}(I)=1 \wedge$

$\forall J\left[J \in \mathscr{R}_{I}^{c} \rightarrow \exists e^{\prime}\left[\mathrm{jump}\left(e^{\prime}\right.\right.\right.$, the horse $\left.\left.\left.\left.) \wedge J=\tau\left(e^{\prime}\right)\right]\right]\right]\right]$

One possible solution, adopted for example in Kratzer 2007 and Champollion 2010b, is to exploit a notion familiar from algebraic semantics: event predicates are closed under sum formation (Krifka 1986, 1998). For example, the predicate denoted by jump not only holds of single jumps, but also of sums of jumps. Some of these sums could be hour-long events. But this by itself does not explain why iterative interpretations engender processing cost. Recent self-paced reading studies show that this cost is even observed in the case of durative atelic predicates when they have an iterative reading, as in John swam for a year (Deo, Piñango, Lai \& Foster-Hanson 2012). This is unexpected if swim is closed under sum formation.

Another possible solution is to adopt a silent verb-level ITER operator (e.g., Egg 1995) and to assume that inserting it increases processing cost, e.g., due to backtracking. A popular early implementation is found in Moens \& Steedman 1988. In that implementation, punctual events are mapped to processes via a silent type coercion operator, which we may think of as ITER. However, if the cost of iterative interpretations is attributed to ITER, the (Deo et al. 2012) results suggest that ITER should not only be present on punctual events like jump but even on processes like swim, which already have the right type in that implementation. I conclude that ITER should not be implemented as a type coercion process. This is consistent with the algebraic semantic view, on which the difference between telic and atelic VPs is a difference between predicates and not between types. Here for simplicity I assume that ITER just has the meaning of the star operator (Link 1983), which does not shift types and which, once it has been inserted, has the same semantic effect as closure under sum. Approximately, it means "once or repeatedly". This is shown in (42). 
[[The horse [jump ITER] for an hour $]$ step 1

$=\lambda P \lambda I\left[\exists e{ }^{*}\right.$ jump $(e$, the horse $) \wedge I=\tau(e) \wedge \operatorname{hours}(I)=1 \wedge$

$\forall J\left[J \in \mathscr{R}_{I}^{c} \rightarrow \exists e^{\prime}\left[{ }^{*}\right.\right.$ jump $\left(e^{\prime}\right.$, the horse $\left.\left.\left.\left.) \wedge J=\tau\left(e^{\prime}\right)\right]\right]\right]\right]$

Iterative interpretations of semelfactives typically involve an alternation of times at which the predicate is true and times at which it is false. The star operator does not semantically entail this alternation, because sum events with continuous runtimes fall under the denotation of the event predicate as well as sum events with discontinuous runtimes. This is justified if the alternation is a pragmatic implicature, as opposed to a semantic entailment, as argued by Egg (1995). However, if gaps turn out to be semantic entailments of iterative readings, then an account like van Geenhoven 2004 may be preferable, in which ITER explicitly enforces the presence of gaps, unlike Link's star operator. I come back to ITER in the conclusion, where I briefly address D\&P's arguments against it.

\section{The scopal behavior of indefinites}

In (40), I had added a conjunct that says that the predicate applies at the whole timespan described by the for-adverbial. This section shows how this conjunct accounts for the scopal behavior of indefinites (following ideas in Zucchi \& White 2001; van Geenhoven 2004; Kratzer 2007; Champollion 2010b).

The added conjunct requires predicates such as push a cart and find a flea to hold at the whole hour. As seen in (43), the existential $\exists x$ that corresponds to the indefinite ends up in the underlined part, outside of the scope of $\forall J$. The underlined part in (43) requires that a single cart be pushed over the course of the hour $I$. This feature of the analysis is shared by the classical account found for example in Krifka 1986, 1998.

[[Mary push a cart for an hour $]]_{\text {step } 1}$

$=\lambda I\left[\forall J\left[J \in \mathscr{R}_{I}^{c} \rightarrow \exists e \exists x[\operatorname{cart}(x) \wedge \operatorname{push}(\operatorname{mary}, e, x) \wedge \tau(e)=J]\right] \wedge \operatorname{hours}(I)=\right.$ $1 \wedge \exists e \exists x[\operatorname{cart}(x) \wedge \operatorname{push}(m, e, x) \wedge \tau(e)=I]]$

When necessary, I assume that ITER is present at V-level, as in the case of iterative interpretations. Thus, (44) is predicted to be true of any five-minute timespan at which there is a number which John dials (once or) repeatedly, and which consists of short subintervals at each of which John dials a number once or repeatedly.

John dialed a wrong phone number for five minutes.

[[John [ITER dial] a number for five minutes $]]_{\text {ste } 1}$

$=\lambda I\left[\exists e \exists x\left[\right.\right.$ number $(x) \wedge^{*} \operatorname{dial}(e$, john, $\left.x) \wedge I=\tau(e)\right] \wedge$ minutes $(I)=5 \wedge$

$\left.\left.\forall J\left[J \overline{\in \mathscr{R}_{I}^{c} \rightarrow \exists e^{\prime} \exists y\left[\operatorname{number}(y) \wedge^{*} \operatorname{dial}\left(e^{\prime}, \mathrm{john}, y\right) \wedge\right.} J=\tau\left(e^{\prime}\right)\right]\right]\right]$ 
The scope and processing of for-adverbials

This "double scope" approach is not yet perfect: it still allows for the possibility that John dials a certain phone number $x$ repeatedly over the course of five minutes (say his wife who he is trying to reach), and at the same time he dials certain other phone numbers $y_{1}, y_{2}, y_{3}$, each of them once (say customers). This can be avoided by adding the requirement that the events whose themes are $y_{1}, y_{2}, y_{3}$ sum up to the event whose theme is $x$. This requires the background assumption that themes are cumulative (that is, the theme of the sum of two events is the sum of their themes), pace Kratzer 2003. See Champollion 2010b for how this can be done. Here I will omit this step in order not to clutter the notation.

I now discuss bare plurals; mass nouns are handled in a parallel way. Bare plurals require a slight change in the analysis, because they can "covary" without special context and will need to be distinguished from indefinites. They take semantic scope above for-adverbials through the same mechanism as indefinites. But their meaning contribution is the same as if they took narrow scope under a universal quantifier. Following van Geenhoven (2004), I assume that the ITER operator can only apply to the verb level, but not to the verb phrase. Assuming that themes are cumulative, [[[ITER dial] numbers]] can hold of a sum of consecutive dialing events in each of which a number is dialed once or repeatedly. These numbers can differ from event to event. We have seen bare plurals do not require a special context in order to be interpretable in sentences with for-adverbials. I conclude that the value of the partition measure in a for-adverbial is not retrieved anaphorically. As in Champollion 2010b, I assume instead that the partition measure is determined by a vague but not anaphoric predicate "short" which maps any time interval $I$ to a predicate of time intervals which are very short in comparison to $I$ (46). To give some arbitrary examples, short might map a one-hour long interval to the predicate $\lambda I$.minutes $(I) \leq 3$, a one-hundred-year interval to $\lambda I$.months $(I) \leq 5$, and so on.

$$
\begin{aligned}
& [\text { for an hour }]]_{\text {final }} \\
& =\lambda P \lambda I\left[\operatorname{AT}(P, I) \wedge \operatorname{hours}(I)=1 \wedge \forall J\left[J \in \mathscr{R}_{I}^{\text {short }(I)} \rightarrow \operatorname{AT}(P, J)\right]\right]
\end{aligned}
$$

Given this, I predict (47) to be true of any five-minute timespan at which there is a sum of one or more numbers which John dials (once or) repeatedly, and which consists of - relatively speaking - very short subintervals, at each of which there is a sum of one or more numbers which John dials. Thus the numbers may vary.

$$
\begin{aligned}
& {\left[[\text { John }[\text { ITER dial] numbers for five minutes }]]_{\text {final }}\right.} \\
& =\lambda I[\exists e[[[\text { John }[\text { ITER dial] numbers }]](e) \wedge I=\tau(e)] \wedge \text { minutes }(I)=5 \wedge \\
& \left.\forall J\left[J \in \mathscr{R}_{I}^{\text {short }(I)} \rightarrow \exists e^{\prime}\left[[[\text { John }[\mathrm{ITER} \text { dial }] \text { numbers }]]\left(e^{\prime}\right) \wedge J=\tau\left(e^{\prime}\right)\right]\right]\right] \\
& =\lambda I\left[\exists e \exists X{ }^{*} \text { number }(X) \wedge^{*} \operatorname{dial}(e, \text { john }, X) \wedge I=\tau(e)\right] \wedge \operatorname{minutes}(I)=5 \wedge \\
& \left.\forall J\left[J \in \mathscr{R}_{I}^{\text {short }(I)} \rightarrow \exists e^{\prime} \exists Y\left[{ }^{*} \text { number }(Y) \wedge{ }^{*} \operatorname{dial}\left(e^{\prime}, \text { john }, Y\right) \wedge J=\tau\left(e^{\prime}\right)\right]\right]\right]
\end{aligned}
$$


Finally, let me sketch an account of salience and world knowledge effects. More details and a clean implementation within a general theory of distributivity can be found in Champollion 2010b.

Recall that singular indefinites take longer to read than bare plurals and require support from a contextually salient temporal predicate like daily or yearly. In Champollion 2010b I have argued that this should be subsumed under the more general phenomenon of nonatomic distributivity discussed for example in Gillon 1990, Schwarzschild 1996, and elsewhere. Normally, in order to distribute an entire VP over nonatomic entities one needs a level of granularity that is salient through context or world knowledge (Lasersohn 1995), as shown in (48). But as Link (1997) points out, a VP whose object is a bare plural is exempt from this requirement, as shown in (49).

a. The men weigh 250 pounds. *per pair

b. (In front of a store window display:) The shoes cost $\$ 50$. $\checkmark$ per pair

a. Rodgers, Hammerstein and Hart wrote a musical. *pairwise

b. Rodgers, Hammerstein, and Hart wrote musicals. $\quad \checkmark$ pairwise

Following Schwarzschild (1996) and Champollion (2010b), I model this context dependency by assuming that there is a VP-level D operator that contains an anaphoric cover over contextually salient entities (pairs of shoes, intervals, etc.).

The shoes [D [cost \$50]].

$\approx$ Each contextually salient set of shoes costs $\$ 50$.

$\approx$ e.g., Each pair of shoes costs $\$ 50$.

nonatomic distributive

Here, for ease of comparison, I use D\&P's notion of a regular partition $\mathscr{R}_{I}^{C}$ to play the role of Schwarzschild's anaphoric covers. That is, I assume that the predicate $C$ in Schwarzschild's D operator is anaphoric on a salient partition. Here is my entry of the temporal version of the D operator. The superscripted $\tau$ is a mnemonic reminder for temporal; the subscripted $C$ is a free variable that is resolved anaphorically by the context.

$$
\left[\left[\mathrm{D}_{C}^{\tau}\right]\right]=\lambda P \lambda I \forall J\left[J \in \mathscr{R}_{I}^{C} \rightarrow \operatorname{AT}(P, J)\right]
$$

This temporal distributivity operator roughly means "at every contextually given interval". Depending on context, it might be interpreted as "every day" or "every winter", for example.

Given this entry, the following sentence is predicted to be true of any interval that lasts one month and which is such that at every cell of its contextually determined regular partition, John takes two pills: 
The scope and processing of for-adverbials

$\left[\left[\text { John } D_{C}^{\tau} \text { [take two pills] for a month }\right]\right]_{\text {final }}$ $=\lambda I\left[\operatorname{months}(I)=1 \wedge \forall J\left[J \in \mathscr{R}_{I}^{C} \rightarrow \exists e[[[\right.\right.$ John take two pills $]](e) \wedge J=$ $\tau(e)] \wedge \forall J\left[J \in \mathscr{R}_{I}^{\text {short }(I)} \rightarrow \exists e^{\prime}\left[\left[\left[\right.\right.\right.\right.$ John $D_{C}^{\tau}[$ take two pills $\left.\left.\left.\left.\left.]\right]\right]\left(e^{\prime}\right) \wedge J=\tau\left(e^{\prime}\right)\right]\right]\right]$

By putting the anaphoricity into the D operator rather than the for-adverbial, I have conditioned the anaphoricity indirectly on the algebraic properties of the VP. A VP with a bare plural object will already be temporally nonquantized and will therefore not need the D operator, so it will not be anaphoric on context. By contrast, a VP with a singular indefinite may require the $\mathrm{D}$ operator in order to become temporally nonquantized. Retrieval of an antecedent for the anaphoric variable $C$ in this D operator will lead to higher processing load.

Finally, a brief comment about partitive interpretations such as polish the countertop. Recall that these interpretations can be blocked by an explicit endpoint description like smooth or to a shine. We need a theory in which polish the countertop smooth/to a shine is temporally quantized, but polish the countertop is not. On closer inspection, polish the countertop is an example of variable telicity:

Mary polished the countertop for/in 15 minutes.

So it should come out as being temporally quantized in some contexts but not in all. The theory of variable telicity in Kennedy 2012 provides such an account. This theory assumes a parametrized measure function partof, which provides a measure of the degree to which a quantity constitutes a material part of an individual (such as a countertop) in a given event. When there is no overt expression like to a shine that could saturate the degree argument of that measure function, it is set to an appropriate standard of comparison, which can be either maximum or minimum. This ambiguity is independently needed for degree achievements and gradable adjectives, and in fact the theory was originally developed for these cases. As a result, a verb phrase like polish the countertop is ambiguous between the following two representations:

[[polish the countertop $]]=$

a. $\quad \lambda e . \exists x[\operatorname{polish}(e) \wedge \operatorname{partof}($ countertop $)(x)(e)=1]$

b. $\quad \lambda e . \exists x[\operatorname{polish}(e) \wedge \operatorname{partof}(\operatorname{countertop})(x)(e)>0]$

The predicate in (54a) is true of any polishing event in which the entire countertop is affected; the predicate in (54b) is true of any polishing event in which some of the countertop is affected. Given appropriate background assumptions about polishing, the former predicate is temporally quantized but the latter predicate is not. 


\section{Summary and outlook}

To summarize, this paper aims to formulate the exact truth conditions of for-adverbials by synthesizing the account of Deo \& Piñango (2011) with more traditional accounts which treat the unacceptability of for-adverbials that modify telic predicates as a semantic rather than a pragmatic phenomenon. This provides a point of interaction between for-adverbials and algebraic semantic theories of aspectual composition as in Krifka 1998. Since atelicity is often operationally defined as compatibility with for-adverbials, the exact truth conditions of these adverbials bear on the question of what exactly — formally speaking — atelicity is.

The proposal described here has broader implications for the formal correlates of processing difficulties, the relation between iterativity and temporal distributivity, the plausibility of the lexical cumulativity hypothesis, and the representation of other aspectually sensitive phenomena such as generics. Let me briefly comment on these points.

D\&P's review of the psycholinguistic literature shows that iterative interpretations are associated with higher processing cost by various measures. D\&P attribute this cost to the process of anaphoric retrieval, and they locate this process in the for-adverbial. In the system presented here, higher processing cost may in principle come from two sources: backtracking in order to insert the V-level iterativity operator whenever there is an iterative reading, and anaphoric retrieval due to the VP-level distributivity operator that is present whenever there is an unexpected covariation of an indefinite due to context. Let me briefly explain why I think we need two operators. Since the latter operator is only needed to "repair" quantized VPs, I expect that iterative predicates derived from quantized VPs should have a higher processing cost than those derived from nonquantized VPs. This prediction seems correct, as we have seen in connection with example (18a). Extrapolating from this example, it seems that the processing cost of iterativity is not uniform but varies according to the algebraic properties of the underlying predicates. This would be mysterious under any account that does not pay attention to these properties. On the other hand, iterativity itself always increases the processing load. As mentioned, the studied by (Deo et al. 2012) show that iterativity leads to higher processing cost even in the case of atelic predicates when they have an iterative reading, as in John swam for a year. I take this as an indication that we need both the VP-level distributivity operator and the verb-level iterativity operator.

The idea that ITER, in this case, is essentially doing the work of lexical cumulativity is incompatible with the lexical cumulativity assumption, which would make ITER redundant here. That is, the added processing would be unexpected here if swim was closed under sum formation, as lexical cumulativity assumes. But at the same time it seems clear that we need at least some restricted version of lexical 
The scope and processing of for-adverbials

cumulativity, in order to get cumulative inferences like the one shown below (Krifka 1992). I leave the question of how to restrict lexical cumulativity to further work.

a. John swam to Dover and Bill swam to Folkestone.

b. $\Rightarrow$ John and Bill swam to Dover and to Folkestone.

The assumption of ITER raises more questions. Plausibility evidence for the presence of ITER is available from languages in which it is overt, e.g., West Greenlandic (van Geenhoven 2004). D\&P reject an ITER-based approach in part because iterative readings of semelfactives occur with higher frequency, an observation they say "has remained in the background in the literature". For this reason, they expect that ITER should become conventionalized and not lead to higher costs. If this observation is correct, an explanation is needed why ITER does not get conventionalized or why this process does not reduce its costs.

D\&P also observe that the experimental correlates of iterative reinterpretation look like a process that gradually emerges and then slowly tapers off, and not like the discrete "fix" that they say one might expect on an ITER-based approach as advocated here. However, it is not clear if the process of anaphoric partition retrieval that D\&P assume is a better or worse match for the continuous nature of this observation than the operator-based approach presented here. To bridge the gap between formal semantic theories and processing observations, additional assumptions are needed that map certain kinds of operations to discrete events and others to continuous processes. On a computer, both the insertion of an operator into the parse tree and the instantiation of a free variable with a value would typically be implemented as discrete events. But in the human brain, we might expect both kinds of operations to be realized as continuous processes.

Looking beyond for-adverbials, D\&P strive for theoretical parsimony by deriving their account of for-adverbials from a more general theory of imperfective and generic/habitual sentences proposed in Deo 2009 for English and Gujarati. Habitual sentences show analogous scopal effects to for-adverbials (Rimell 2004; Krifka, Pelletier, Carlson, ter Meulen, Chierchia \& Link 1995: 39f.).

a. Mary smokes cigarettes / *a cigarette.

b. Mary smokes cigarettes / a cigarette after dinner.

a. Yesterday, Mary smoked cigarettes / *a cigarette for an hour.

b. Last month, Mary smoked a cigarette after dinner for a week.

Similar effects both for habituals and for for-adverbials hold in Hindi (Deo, p.c.), which is close to Gujarati. This calls for propagating the changes to D\&P proposed here to the theory of habituals in Deo 2009. 
Lucas Champollion

\section{References}

Bennett, Michael R. \& Barbara H. Partee. 1972. Toward the Logic of Tense and Aspect in English. Indiana University Linguistics Club.

Brennan, James \& Liina Pylkkänen. 2008. Processing events: Behavioral and neuromagnetic correlates of aspectual coercion. Brain and Language 106(2). 132-143. doi:10.1016/j.bandl.2008.04.003.

Carlson, Gregory N. 1977. Reference to kinds in English. Amherst, MA: University of Massachusetts dissertation.

Champollion, Lucas. 2010a. For-adverbials and the specified quantity generalization. Presented at the $34^{\text {th }}$ Penn Linguistics Colloquium (PLC), University of Pennsylvania.

Champollion, Lucas. 2010b. Parts of a whole: distributivity as a bridge between aspect and measurement. Philadelphia, PA: University of Pennsylvania dissertation.

Deo, Ashwini. 2009. Unifying the imperfective and the progressive: Partitions as quantificational domains. Linguistics and Philosophy 32(5). 475-521. doi:10.1007/s10988-010-9068-z.

Deo, Ashwini \& Maria Mercedes Piñango. 2011. Quantification and context in measure adverbs. In Twenty-first Conference on Semantics and Linguistic Theory (SALT), vol. 21, 295-312.

Deo, Ashwini, Maria Mercedes Piñango, Yao-Ying Lai \& Emily Foster-Hanson. 2012. Building multiple events: the cost of context retrieval. Presentation at Architectures and Mechanisms for Language Processing 2012 (AMLaP).

Downey, Ryan Andrew. 2006. Examination of lexical properties during auditory sentence processing using event-related potentials: University of California, San Diego and San Diego State University dissertation.

Dowty, David R. 1979. Word meaning and Montague grammar. Dordrecht, Netherlands: Reidel.

Egg, Markus. 1994. Aktionsart und Kompositionalität. Berlin, Germany: Akademie Verlag.

Egg, Markus. 1995. Aspect and quantification - an iterative approach. In Tenth Amsterdam Colloquium, Amsterdam, Netherlands: ILLC.

van Geenhoven, Veerle. 2004. For-adverbials, frequentative aspect, and pluractionality. Natural Language Semantics 12. 135-190. doi:10.1023/B:NALS.0000031389.36427.af.

van Geenhoven, Veerle. 2005. Atelicity, pluractionality and adverbial quantification. In H. J. Verkuyl, H. de Swart \& A. van Hout (eds.), Perspectives on Aspect, 107-124. Berlin, Germany: Springer. doi:10.1007/1-4020-3232-3_6.

Gillon, Brendan S. 1990. Plural noun phrases and their readings: A reply to 
The scope and processing of for-adverbials

Lasersohn. Linguistics and Philosophy 13. 477-485. doi:10.1007/BF00630751. Kennedy, Chris. 2012. The composition of incremental change. In Violeta Demonte \& Louise McNally (eds.), Telicity, Change, and State: A Crosscategorial View of Event Structure, Oxford, UK: Oxford University Press. doi:10.1093/acprof:oso/9780199693498.003.0004.

Kratzer, Angelika. 2003. The Event Argument and the Semantics of Verbs, Chapter 3. Manuscript. Amherst: University of Massachusetts, Amherst, MA.

Kratzer, Angelika. 2007. On the plurality of verbs. In Johannes Dölling, Tatjana Heyde-Zybatow \& Martin Schäfer (eds.), Event Structures in Linguistic Form and Interpretation, 269-300. Berlin, Germany: de Gruyter.

Krifka, Manfred. 1986. Nominalreferenz und Zeitkonstitution. Zur Semantik von Massentermen, Pluraltermen und Aspektklassen. Munich, Germany (published 1989): Fink.

Krifka, Manfred. 1992. Thematic relations as links between nominal reference and temporal constitution. In Ivan A. Sag \& Anna Szabolcsi (eds.), Lexical Matters, 29-53. Stanford, CA: CSLI Publications.

Krifka, Manfred. 1998. The origins of telicity. In Susan Rothstein (ed.), Events and Grammar, 197-235. Dordrecht, Netherlands: Kluwer.

Krifka, Manfred, Francis Jeffry Pelletier, Gregory N. Carlson, Alice ter Meulen, Gennaro Chierchia \& Godehard Link. 1995. Genericity: An introduction. In Gregory N. Carlson \& Francis Jeffry Pelletier (eds.), The Generic Book, 1-124. Chicago, IL: University of Chicago Press.

Lasersohn, Peter. 1995. Plurality, Conjunction and Events. Dordrecht, Netherlands: Kluwer.

Link, Godehard. 1983. The logical analysis of plurals and mass terms: A latticetheoretical approach. In Reiner Bäuerle, Christoph Schwarze \& Arnim von Stechow (eds.), Meaning, Use and Interpretation of Language, 303-323. Berlin, Germany: de Gruyter.

Link, Godehard. 1997. Ten years of research on plurals — where do we stand? In Fritz Hamm \& Erhard Hinrichs (eds.), Plurality and Quantification, 19-54. Dordrecht, Netherlands: Kluwer.

Moens, Marc \& Mark Steedman. 1988. Temporal ontology and temporal reference. Computational Linguistics 14(2). 15-28.

Moltmann, Friederike. 1991. Measure adverbials. Linguistics and Philosophy 14. 629-660. doi:10.1007/BF00631962.

Piñango, Maria Mercedes, Aaron Winnick, Rashad Ullah \& Edgar Zurif. 2006. Time-course of semantic composition: The case of aspectual coercion. Journal of Psycholinguistic Research 35(3). 233-244. doi:10.1007/s10936-006-9013-z.

Piñango, Maria Mercedes \& Edgar Zurif. 2001. Semantic operations in aphasic comprehension: Implications for the cortical organization of language. Brain 
and Language 79(2). 297-308. doi:10.1006/brln.2001.2492.

Piñango, Maria Mercedes, Edgar Zurif \& Ray S. Jackendoff. 1999. Realtime processing implications of enriched composition at the syntaxsemantics interface. Journal of Psycholinguistic Research 28(4). 395-414. doi:10.1023/A:1023241115818.

Piñón, Christopher. 1999. Durative adverbials for result states. In Eighteenth West Coast Conference on Formal Linguistics (WCCFL), 420-433.

Rimell, Laura. 2004. Habitual sentences and generic quantification. In G. Garding \& M. Tsujimura (eds.), Twenty-third West Coast Conference on Formal Linguistics (WCCFL), Somerville, MA: Cascadilla Press.

Schwarzschild, Roger. 1996. Pluralities. Dordrecht, Netherlands: Kluwer.

Smith, Carlota. 1997. The Parameter of Aspect (second edition). Dordrecht, Netherlands: Reidel.

Smollett, Rebecca. 2005. Quantized direct objects don't delimit after all. In Henk J. Verkuyl, Henriëtte de Swart \& Angeliek van Hout (eds.), Perspectives on Aspect, 41-59. Berlin, Germany: Springer. doi:10.1007/1-4020-3232-3_3.

Todorova, Marina, Karen Straub, William Badecker \& Robert Frank. 2000. Aspectual coercion and the online computation of sentential aspect. Twenty-Second Annual Conference of the Cognitive Science Society (CSS) 3-8.

Vendler, Zeno. 1957. Verbs and times. The Philosophical Review 66. 143-160.

Verkuyl, Henk J. 1972. On the Compositional Nature of the Aspects. Dordrecht, Netherlands: Reidel.

Verkuyl, Henk J. 1989. Aspectual classes and aspectual composition. Linguistics and Philosophy 12(1). 39-94. doi:10.1007/BF00627398.

Zucchi, Sandro \& Michael White. 2001. Twigs, sequences and the temporal constitution of predicates. Linguistics and Philosophy 24. 187-222. doi:10.1023/A:1005690022190.

Lucas Champollion

Department of Linguistics

New York University

10 Washington Place

New York, NY 10003

champollion@nyu.edu 